\title{
Freeze-fracture replica immunogold labeling (FRIL) in biological electron microscopy
}

\author{
J. E. Rash, ${ }^{*}$ K. G. V. Davidson, ${ }^{*}$ N. Kamasawa, ${ }^{*}$ and T. Yasumura, ${ }^{*}$ \\ *Department of Biomedical Sciences, Colorado State University, Fort Collins, CO 80523
}

Fujimoto [1] developed "SDS-FRL", the first practical method for labeling membrane proteins in freeze-fracture replicas. He recognized that freeze-fracture replicas, made by vapor deposition of platinum and carbon onto freshly cleaved surfaces under high vacuum, are equivalent to "finelydivided" platinum and "activated carbon", which are highly adsorptive [2]. Washing with SDS detergent removed bulk tissue components from the replicas, leaving molecules that were directly adsorbed to the replicas available for labeling. Because vigorous washing of unsupported replicas destroyed replica integrity, and because histological mapping of neuronal and glia processes in brain and spinal cord requires maintained tissue integrity, we combined "confocal grid-mapped freeze fracture" [3] with SDS-FRL [1] in a hybrid technique that we call FRIL [4] to indicate its capability for mapping immunogold-labeled membrane proteins from the gross anatomical level to the ultrastructural level. Because most cytoplasmic processes in the central nervous system are smaller than the limit of resolution of light microscopy, it is impossible to map proteins to individual neuronal and glial processes by confocal microscopy, and many of the components (e.g., neuronal gap junctions) are too rare to find statistically-relevant numbers by conventional TEM immunocytochemistry. However, freeze-fracture replicas provide large expanses of membranes, suitable for finding intramembrane particle arrays; and by FRIL, high-visibility immunogold beads provide the "neon signs" that call attention to their presence (Fig. 1A-C).

FRIL is straightforward but very time-consuming. It takes five days to prepare one group of samples: one day for sample fixation, Vibratome sectioning to uniform sample thickness, and freezing; one day for freeze-fracturing, attaching a gold "index" grid to the frozen sample in a thin coat of Lexan plastic, and air-drying at $-35^{\circ} \mathrm{C}$; one day for photomapping and SDS/collagenase cleaning; one day for labeling using monoclonal and polyclonal antibodies, followed by secondary immunogold labeling for the several species of primary antibodies (Fig. 2); and one day for rinsing, air drying, carbon coating and removal of the Lexan support film. Because each replica is ca. $4,000,000 \mu \mathrm{m}^{2}$ (Fig. 3), of which ca. $1,500,000 \mu \mathrm{m}^{2}$ is potentially examinable - at $1 \mu \mathrm{m}^{2} / \mathrm{sec}-$ detailed confocal maps obtained before tissue washing reduce the time for preliminary examination from $300 \mathrm{hrs}$ of beam time per sample to a few hours. The initial evaluative step is to ascertain the level of non-specific "background". If background labeling is $>10$ gold beads $/ \mu \mathrm{m}^{2}$, we discard the replicas. With good "blocking buffers" [2], non-specific background is reduced to $<1$ gold bead/ $\mu \mathrm{m}^{2}$ (usually $<0.1 / \mu \mathrm{m}^{2}$ ), whereas specific labeling may be $>300 / \mu \mathrm{m}^{2}$ for a "signal-to-noise ratio" $>3000: 1$. Using FRIL, we have identified a variety of transmembrane proteins in nerve and glial cell processes [5,6], including 10 different connexins, several different glutamate receptors (Fig. 1B-C), a GABA receptor, aquaporin4 [4], and zonula occludens-1 [7].

The limit of resolution of confocal microscopy (ca. $1 / 4 \mu \mathrm{m}$ ) means that clusters of labeled proteins cannot be discriminated from individual sources of noise ("false positives") that may arise from clumping of primary or secondary antibodies. FRIL allows high-resolution visualization of specific molecular arrays; and specificity of labeling is confirmed when only that particular class of protein is labeled. Nevertheless, FRIL has its limitations: a) currently, only transmembrane proteins and tightly-bound accessory proteins remain after SDS washing to permit immunogold labeling [7]; 
b) some transmembrane proteins are removed by excessive washing, resulting in "false negatives"; and c) either the extraplasmic or protoplasmic face of each transmembrane protein is coated with platinum, thereby making that side of the protein unavailable for labeling. Consequently, antibody labels must be carefully designed to label either extracellular or intercellular epitopes for each target protein. Despite these caveats, FRIL has become an essential technique for identifying clustered transmembrane proteins in animals (references cited above), plants [8], and bacteria [9].

\section{References}

[1] K. Fujimoto Histochem. Cell Biol. 107(1997) 87.

[2] J. E. Rash et al., Freeze-Fracture Studies of Membranes, CRC Press, Boca Raton, 1990.

[3] J. E. Rash et al., Techniques in Modern Biomedical Microscopy. Vol 2. Rapid Freezing, Freezefracture and Deep Etching, Wiley-Liss, 1995.

[4] J. E. Rash and T. Yasumura, Cell Tissue Res. 296 (1999) 307.

[5] J. E. Rash et al. J. Neurosci. 21 (2001) 1983.

[6] A. Pereda et al., J. Neurosci. 23 (2003) 7489.

[7] J. E. Rash et al., J. Neurocytol. 33 (2004) 131.

[8] S. Kimura et al., Plant Cell 11 (1999) 2075.

[9] S. Kimura et al., J. Bacteriol. 183 (2001) 5668.

[10] Supported by NIH grants NS44010, NS44395, and NS38121 (JER).
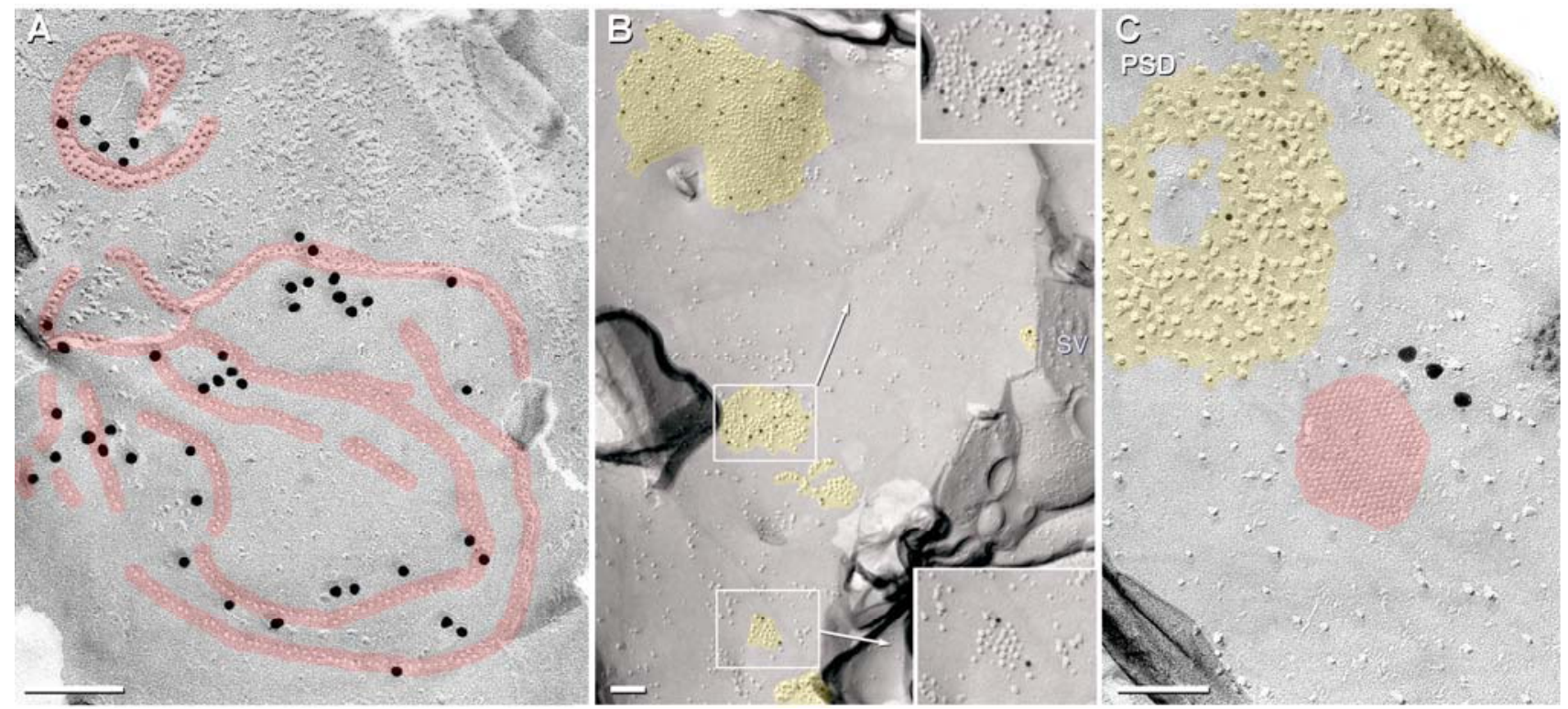

Fig. 1. Axon terminals in rat retina $(A)$ and hippocampus $(B)$ and goldfish brain $(C)$ immunogold labeled for connexin36 $(\mathrm{A}, \mathrm{C})$ and NR1 glutamate receptors $(B, C)$. Scale bars $=0.1 \mu \mathrm{m}$.
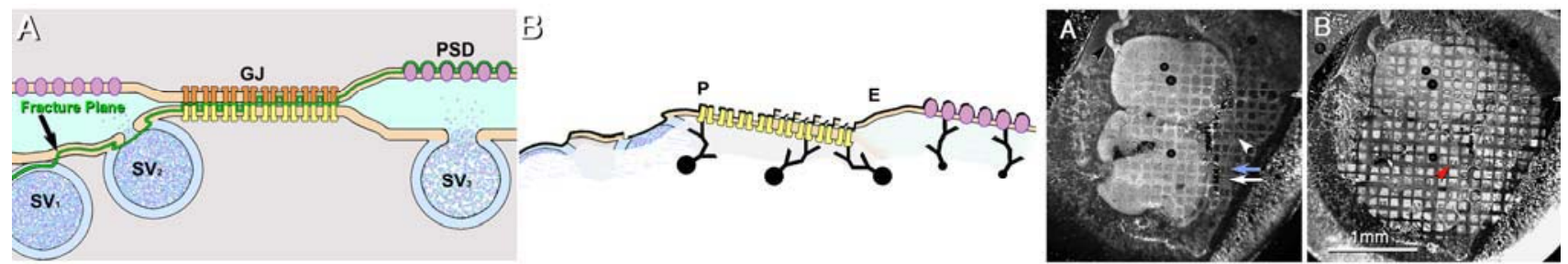

Fig. 2. Diagram illustrating two sizes of immunogold beads on glutamate receptors and connexin 36 proteins remaining after SDS. Fig. 3. Confocal map, postnatal rat spinal cord before SDS washing. 